\title{
Savoir manger (et boire)
}

\section{Hans Stalder}

Prof. Dr méd., membre de la rédaction

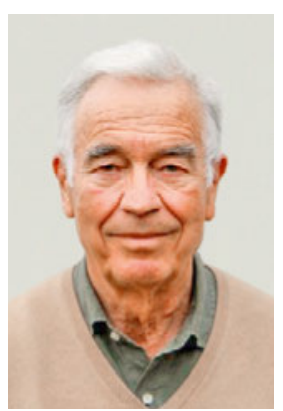

Quand j'étais assistant - ça fait un moment - la diététique jouait un grand rôle. A défaut d'endoscopie, le professeur de gastroentérologie dirigeait l'école de diététique et prêchait des régimes sévères: la diète Sippy 1,2 et 3 , qui consistait en une alimentation des plus fades, avec des produits laitiers pour les ulcères, un régime sans sel strict pour les insuffisances cardiaques, sans graisses pour les cholécystites et à zéro calorie pour les obèses. Plus tard, après l'introduction de médicaments puissants, les hôpitaux s'ingénièrent plutôt à proposer de bons repas.

Dans les années 70, le culte des régimes est réapparu, mais plutôt dans le domaine de la santé publique. Après une étude de cohorte multinationale qui montrait que la consommation des graisses saturées était associée à l'artériosclérose, et donc aux maladies cardiovasculaires, le monde entier se sentit obligé de diminuer les graisses et remplacer la perte d'énergie par des carbohydrates. Pas d'œufs non plus, car ils contiennent du cholestérol! Mais si ces auteurs avaient inclus la France dans leur étude, ils auraient découvert le french miracle, soit qu'on peut très bien manger des graisses sans mourir davantage de maladies cardiovasculaires. Ainsi, le régime méditerranéen était né.

Et aujourd'hui? Selon Bertrand Kiefer [2], il y a à nouveau un "renversement des certitudes»: une nouvelle étude, faite par plus de 200 investigateurs, impliquant plus de 133000 personnes dans 18 pays, démontre que les maladies cardiovasculaires sont associées à la consommation d'hydrates de carbone [3]. Manger des graisses animales serait donc sain! Et déjà l'hypothèse est proposée que le changement des graisses aux hydrates de carbone durant ces dernières décennies nous aurait rendus obèses [1]...

Idem pour les boissons alcooliques: longtemps, on nous a enseigné que boire modérément de l'alcool était bénéfique pour la santé (grâce à la célèbre courbe en J). Or un article récent montre que même de petites quantités d'alcool provoquent des altérations anatomiques et physiologiques du cerveau [4].

Comment est-on arrivé là? L'explication me semble simple: toutes ces recommandations sont basées sur des études de cohortes. Dans le domaine de la prévention primaire par l'alimentation, il n'y a que peu d'études prospectives et randomisées, et de toute façon pas à double insu (ce qui n'est pas possible dans ce domaine). Or on sait au moins depuis la révision des recommandations pour le traitement hormonal postménopausique qu'il faut être très prudent en propageant des recommandations se basant uniquement sur des études de cohortes. Ces incertitudes ont permis la pullulation de propositions de régimes n'ayant aucune base scientifique - végétalien, végan, sans gluten, bio -, qui remplissent les rayons des librairies et sont diffusées sur Internet au profit de forts lobbies alimentaires.

Une des seules études randomisées et prospectives a été faite en Espagne. Elle a montré que l'adjonction d'huile d'olive ou de noix diminue l'incidence de maladies cardiovasculaires chez des personnes à risque [5]. Mais attention: avant d'ingérer noix et huile d'olives, rappelons que nous ne sommes pas tous des personnes à risque ni des Espagnols!

Que faut-il recommander alors? Que nos collègues préventologues de la santé publique soient prudents avec leurs recommandations [1]! Puis il faut noter que les enquêtes [3, 4] qui «renversent des certitudes» [2] ne sont également que des études de cohortes. Donc il n'y a pas de raison de s'affoler. Le côté positif est que ces résultats contradictoires et le manque d'études prospectives et randomisées nous permettent au moins de consommer en toute quiétude les plats et boissons que nous aimons. Mais aussi de retenir le principe du vieux Jean Anthelme Brillat-Savarin (plus connu pour sa phrase «Dis-moi ce que tu manges: je te dirai qui tu es»), qui constate dans son livre «Physiologie du goût, ou méditations de gastronomie transcendante» que "Ceux qui s'indigèrent ou qui s'enivrent ne savent ni boire ni manger» [6]. Et si vous (ou vos malades) êtes obèses? Alors là il n'y a qu'à suivre un régime qui, comme ceux d'antan, est fort désagréable: FdH («Friss die Hälfte»): bouffe la moitié!

\section{Références}

1 Nissen SE. U.S. dietary guidelines: an evidence-free zone. Ann Intern Med. 2016;164:558-9.

2 Kiefer B. Nutrition: le renversement des certitudes. Revue médicale suisse. 2017;13:1632.

3 Dehghan M, Mente A, Zhang X, et al. Prospective Urban Rural Epidemiology (PURE) study investigators. Associations of fats and carbohydrate intake with cardiovascular disease and mortality in 18 countries from five continents (PURE): a prospective cohort study. Lancet. 2017 Aug 28. pii: S0140-6736(17)32252-3. doi: 10.1016/S0140-6736(17)32252-3.

4 Topiwala A, Allan CL, Valkanova V, et al. Moderate alcohol consumption as risk factor for adverse brain outcomes and cognitive decline: longitudinal cohort study. BMJ. 2017;357:j2353. doi: 10.1136/bmj.j2353.

5 Estruch RI, Ros E, Salas-Salvadó J, Covas MI, et al. Primary prevention of cardiovascular disease with a Mediterranean diet. $\mathrm{N}$ Engl J Med. 2013;368:1279-90.

6 Brillat-Savarin JA. Physiologie du goût, ou méditations de gastronomie transcendante; ouvrage théorique, historique et à l'ordre du jour, dédié aux gastronomes parisiens, par un professeur, membre de plusieurs sociétés littéraires et savantes. 1825. A. Sautelet. Gabriel de Gosset éd. Paris. 